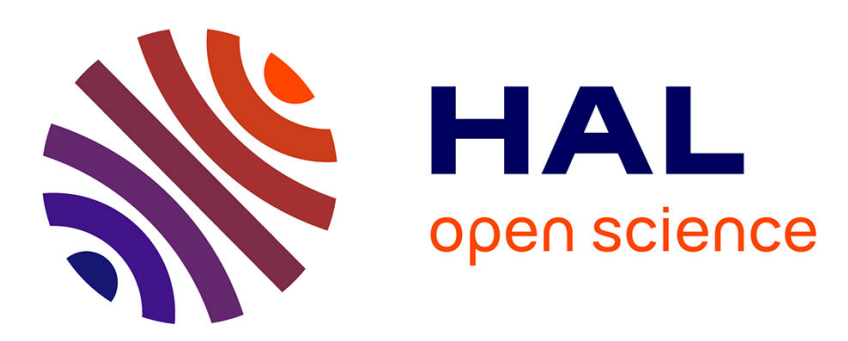

\title{
Design and Implementation of a Predictive Control Strategy for Power Management of a Wireless Sensor Network
}

Olesia Mokrenko, Maria Isabel Vergara Gallego, Warody Lombardi, Suzanne Lesecq, Diego Puschini, Carolina Albea-Sanchez

\section{To cite this version:}

Olesia Mokrenko, Maria Isabel Vergara Gallego, Warody Lombardi, Suzanne Lesecq, Diego Puschini, et al.. Design and Implementation of a Predictive Control Strategy for Power Management of a Wireless Sensor Network. IEEE European Control Conference, Jul 2015, Linz, Austria. hal-01140750

\author{
HAL Id: hal-01140750 \\ https://hal.science/hal-01140750
}

Submitted on 9 Apr 2015

HAL is a multi-disciplinary open access archive for the deposit and dissemination of scientific research documents, whether they are published or not. The documents may come from teaching and research institutions in France or abroad, or from public or private research centers.
L'archive ouverte pluridisciplinaire HAL, est destinée au dépôt et à la diffusion de documents scientifiques de niveau recherche, publiés ou non, émanant des établissements d'enseignement et de recherche français ou étrangers, des laboratoires publics ou privés. 


\title{
Design and Implementation of a Predictive Control Strategy for Power Management of a Wireless Sensor Network
}

\author{
Olesia Mokrenko ${ }^{1}$, Maria Isabel Vergara-Gallego ${ }^{1}$, Warody Lombardi ${ }^{1}$, Suzanne Lesecq ${ }^{1}$, Diego Puschini ${ }^{1}$, \\ and Carolina Albea ${ }^{2}$
}

\begin{abstract}
Technological advances have made wireless sensor nodes cheap and reliable enough to be brought into various application domains. The limited energy capacity of sensor nodes is the key factor that restricts their lifespan. In this paper, a Predictive Control strategy for Dynamic Power Management of a set of wireless sensor nodes is proposed. The control formulation is based on Model Predictive Control with constraints and binary optimization variables, leading to a Mixed Integer Quadratic Programming problem. The control algorithm proposed guarantees services and performances levels with a minimum number of active nodes and/or a minimum load on such components. The strategy is evaluated on a real testbench with wireless sensor nodes equipped with batteries and harvesting systems. Experimental results show the effectiveness of the control method proposed.
\end{abstract}

\section{INTRODUCTION}

Wireless sensor networks (WSNs) consist in a (large) number of sensor nodes (SNs) with sensing, wireless communication and computation capabilities, in order to monitor and/or control the physical world. WSNs have found applications in a wide range of domains, including (among others) structural health monitoring, building automation, military surveillance, and bio-medical health monitoring [1]. Usually, SNs are tiny devices with very limited energy capacity stored in batteries. They may also harvest energy from the environment. These SNs can possess several functioning modes with different capabilities (in terms of e.g. communication and computing) and associated power consumption. Their energy efficiency is a major concern as some application domains advocate for lifespan of at least 10 years without battery replacement [2].

Each SN typically includes four subsystems (SSs) namely, a sensing $S S$ with one or several sensors, a computing $S S$ that provides intelligence (includes Microcontroller Unit (MCU)) to the $\mathrm{SN}$, a communication $S S$ with a radio unit, and a power supply SS, which is the source of energy. It possesses a battery and sometimes a harvesting system (see Figure 1).

The network lifespan is defined as the time interval the network is able to perform the sensing function and to transmit data to the sink. Various studies have been conducted to increase the WSN lifespan in different levels and directions. For instance, at the SN level, [2] and [4] propose to switchoff the $\mathrm{SN}$ or reduce its power consumption when it no

\footnotetext{
${ }^{1}$ Univ. Grenoble Alpes, F-38000 Grenoble, France and CEA, LETI, MINATEC Campus, F-38054 Grenoble, France $\{$ First Name. LastName $\}$ ecea. fr

${ }^{2}$ CNRS, LAAS, 7 avenue du Colonel Roche, F-31400 Toulouse, France and Univ. of Toulouse, UPS, LAAS, F-31400, Toulouse, France calbea@laas. fr
}

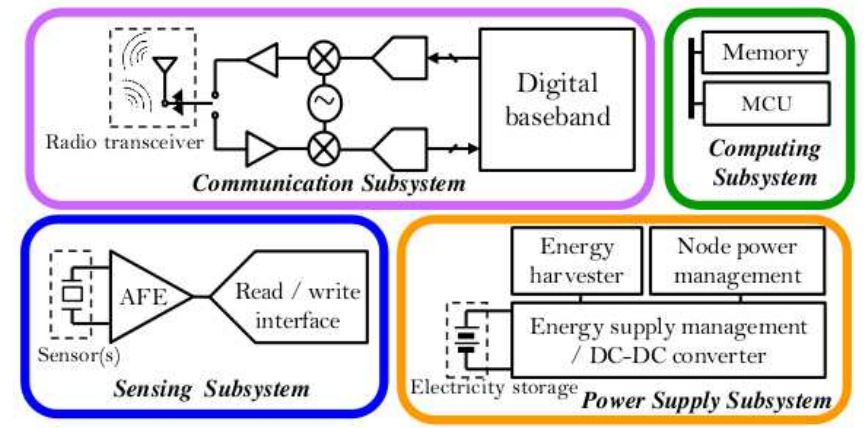

Fig. 1: Functional subsystems of a wireless SN (modified from [3])

longer performs useful tasks, therefore increasing lifespan. Indeed, supplying nodes by the batteries is certainly a major obstacle to a wide public acceptance of WSN because the $\mathrm{SN}$ lifespan is not as expected. Moreover, this may influence the reliability of the application (monitoring and/or control) built on top of the WSN. As a consequence, nodes have to be designed with stringent power consumption constraints [1] and equipped, when possible, with energy harvesting sources [5]. However if each SS in the SN is designed to be power efficient, their association does not necessarily lead to a lowpower SN. Therefore, a power/energy management policy for the whole $\mathrm{SN}$ is mandatory. Note that most of these policies suppose that the sensing SS consumes significantly less energy than the communication SS (especially in transmission mode). However, when "energy-hungry" sensors [6] are embedded, an efficient energy management policy must be implemented to ensure the whole WSN performs the functionality it is supposed to. This global power/energy management policy is certainly a more complex problem than the one dedicated to a single node because, in essence, the WSN is spatially distributed, usually with a clock frequency in each node not (properly) synchronized with the other ones.

Power control in a WSN using multiple-description coding is addressed in [7] and [8]. Their main contribution is to investigate the role of dynamic power control and coding when state estimation is considered. The control objective tries to counteract the channel variability (i.e. ensure Quality of Service (QoS)) and to trade-off battery use for estimation accuracy. The controller is located in a gateway. It decides upon the transmission power level and the coding scheme to be used by each SN. However, satisfying the QoS does not 
guarantee that the "mission" [9] is satisfied.

The main goal of this paper is to extend the lifespan of a WSN by reducing the overall energy consumption of the SNs via an appropriate management of the functioning modes of each $\mathrm{SN}$, in order to provide a given functionality, hereafter named mission, under performance constraints. The mission is expressed as a set of constraints on the different functioning modes of each SN. The power management strategy is applied at the WSN and SN levels. The SNs are equipped with harvesting systems. The control strategy is initially proposed in [10] and extended hereafter to by evaluated on a real test-bench.

In the present work, Model Predictive Control (MPC) is considered as a promising control strategy for power management. It is based on predicting the system trajectories over a receding horizon, while calculating an optimal control policy with respect to a set of constraints [11]. It can be applied to linear Single-Input-Single-Output and MultipleInput-Multiple-Output systems [12], [13], nonlinear [14] and hybrid [15] systems. This last class of systems can present continuous (and sampled) states (real-set variables), discrete and state-machine states (integer-set variables) and logic rules (binary-set variables) [16]. The optimization involved in this case is known as Mixed Integer Programming (MIP) problem.

The paper is organized as follows. Section II is first dedicated to the system modelling. Then the control objectives are provided. The control design is developed in Section III. It is based on Constrained Predictive Control techniques, with bounded states, equality and inequality constraints and binary control values. Section IV implements the proposed control on a real test-bench. Section V summarizes the main results.

Notations: Throughout the paper $\mathbb{N}^{*}$ denotes the set of natural numbers where $\mathbb{N}^{*}=\mathbb{N} \backslash\{0\} . A \in \mathbb{R}^{d \times l}$ is a matrix of size $d \times l$ with real values. $\mathbf{x} \in \mathbb{R}_{+}^{d}$ is a vector of size $d \times 1$ with non-negative real values. $\mathbf{u} \in\{0,1\}^{d l}$ represents the vector $\mathbf{u}$ of dimension $d l \times 1$ whose elements are the binary ( 0 or 1 ) variables. The identity matrix of size $n \times n$ is described by $\mathbf{I}_{n}$, and the null matrix of size $n \times n$ is $\mathbf{0}_{n \times n}$.

\section{System Modeling And Control Objectives}

Consider a WSN with a single-hop heterogeneous sensor network architecture [17] (see Figure 2). The SNs $S_{i}, i=$ $1: n, n \in \mathbb{N}^{*}$ can only communicate with a centralized controller, called sink, which is responsible for monitoring and control of the overall system. All SNs are functionally equivalent: they are interchangeable but their hardware can differ, e.g. batteries, processors may be unalike. Each SN is powered by a battery and may also be equipped with a harvesting source, e.g. a solar cell. SNs can exhibit different functioning modes $M_{j}, j=1: m, m \in \mathbb{N}^{*}$, which are related to the state (on, sleep, off, etc.) of each SN subsystem, characterized by a known power consumption for a given period of time.

Consider that the energy consumption in the WSN is

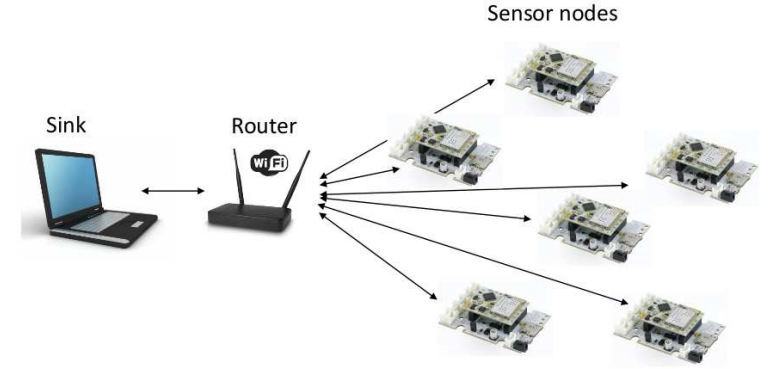

Fig. 2: Hardware architecture

TABLE I: Energy consumption of node $S_{i}, i=1: n$, in the different modes $M_{j}, j=1: m$, over the time period $\Delta$

\begin{tabular}{|l|c|c|c|c|l|}
\hline Sensor node & Mode $M_{1}$ & Mode $M_{2}$ & $\ldots$ & Mode $M_{m}$ & \\
\hline \hline$S_{1}$ & $b_{11}$ & $b_{12}$ & $\ldots$ & $b_{1 m}$ & $=B_{1}$ \\
\hline$\vdots$ & $\vdots$ & $\vdots$ & $\ddots$ & $\vdots$ & $\vdots$ \\
\hline$S_{n}$ & $b_{n 1}$ & $b_{n 2}$ & $\ldots$ & $b_{n m}$ & $=B_{n}$ \\
\hline
\end{tabular}

described by the linear model:

$$
\mathbf{x}_{k+1}=A \mathbf{x}_{k}+B \mathbf{u}_{k}+E \mathbf{w}_{k}
$$

where $\mathbf{x}_{k} \in \mathbb{R}_{+}^{n}$ is the remaining energy in the battery of the SNs at time $k$. The state matrix is $A=\mathbf{I}_{n} \in \mathbb{R}^{n \times n}$. Bu $\mathbf{u}_{k}$ represents the energy that will be consumed during the time interval $[k \Delta,(k+1) \Delta]$, where $\Delta$ is a time period with which the control is done. $E \mathbf{w}_{k}$ corresponds to the energy provided by the harvesting source. The initial battery capacity (i.e. at $k=0$ ) is denoted $\mathbf{x}_{0}$. Notice that for each node $S_{i}$, the energy capacity is constrained:

$$
0 \leqslant x^{i} \leqslant X_{\max }^{i}
$$

$\mathbf{u}_{k}=\left[\mathbf{u}_{1}^{T}, \cdots, \mathbf{u}_{i}^{T}, \cdots, \mathbf{u}_{n}^{T}\right]^{T} \in\{0,1\}^{n m}$ is the control input. Each sub-vector $\mathbf{u}_{i}=\left[u_{i 1}, \cdots, u_{i j}, \cdots, u_{i m}\right]^{T}$ represents the functioning mode of each $S_{i}$, where $u_{i j} \in\{0,1\}$. As each node $S_{i}$ has a unique working mode at time $k$, a set of constraints must be defined:

$$
\forall i=1: n: \sum_{j=1}^{m} u_{i j}=1
$$

The control matrix is $B=\operatorname{diag}\left[-B_{1}, \ldots,-B_{n}\right] \in \mathbb{R}^{n \times n m}$. Each component $b_{i j}$ of $B_{i}$ represents the amount of energy consumed by $S_{i}$ working in mode $M_{j}$ during the time period $\Delta$ (see Table I). Note that a switch from one mode to another one has an extra energy cost that is supposed to be integrated in $b_{i j}$.

The energy recovery element (i.e. harvesting system) $\mathbf{w}_{k} \in$ $\{0,1\}^{n}$ can be seen as a disturbance input that cannot be controlled but may be predicted. Actually, $w_{i}$ corresponds to the ability for node $S_{i}$ to harvest energy. 0 (resp. 1) is associated to the state Off (resp. On) of the harvesting system. $E \in \mathbb{R}^{n \times n}$ is the so-called disturbance matrix:

$$
E=\operatorname{diag}\left[E_{11}, \ldots, E_{n n}\right]
$$


where $E_{i i}$ corresponds to the amount of energy harvested by $S_{i}$ during the period $\Delta$. Note that matrix $E$ is in essence a time-variant matrix in real-life conditions.

\section{Control objectives}

In order to define the control objectives for the system, the mission is introduced. A mission is described by a minimum number $d_{j} \in \mathbb{N}^{*}$ of active SNs (corresponds to the appropriate functioning mode(s) $M_{j}$ ), sufficient to provide the requested services and performance levels. $d_{j}$ may possibly change from time to time. Thus, the mission imposes a new set of constraints:

$$
\sum_{i=1}^{n} u_{i j}=d_{j}
$$

Note that hereafter, this functional constraint is supposed to indirectly ensure the desired QoS for the WSN. Therefore, the system to be controlled is not only constrained by (3), meaning that each node $S_{i}$ is in a unique mode, but also by the set of extra functional constraints (5) that are used to define the mission.

\section{Model Predictive Control Design}

The minimization of the power consumption of (1) can be seen as a Constrained Optimal Control problem that can be described as a Quadratic Programming (QP) problem. Constrained MPC implies the minimization of a cost function based on the predicted system evolution.

Recently, the interest in using MPC for controlling systems that involve a mix of real-valued dynamics and logical rules has arisen [15] [16]. Unfortunately, when this problem is formulated as an optimization one, the resulting description is no longer a QP problem but a Mixed Integer Quadratic Programming (MIQP) problem with two different types of optimization variables, namely, real-valued and binary ones. This makes this latter problem harder to solve when compared to an ordinary QP problem.

It is assumed throughout the rest of the paper that the pair $(A, B)$ in (1) is stabilizable. At each decision time $k \Delta$, the current state (assumed to be available) $\mathbf{x}_{k}=$ $\mathbf{x}_{k \mid k}$ is used to define the optimal control sequence $\mathbf{u}^{*}=$ $\left[\mathbf{u}_{k \mid k}^{T}, \ldots, \mathbf{u}_{k+N_{p}-1 \mid k}^{T}\right]^{T}$ which is the assumed as the minimization problem:

$$
\mathbf{u}^{*}=\arg \min _{u} \sum_{i=0}^{N_{p}-1} \mathbf{x}_{k+i \mid k}^{T} Q \mathbf{x}_{k+i \mid k}+\sum_{i=0}^{N_{u}-1} \mathbf{u}_{k+i \mid k}^{T} R \mathbf{u}_{k+i \mid k}
$$

subject to:

$$
\left\{\begin{array}{l}
\mathbf{x}_{k+i+1 \mid k}=A \mathbf{x}_{k+i \mid k}+B \mathbf{u}_{k+i \mid k}, \quad i=1, \ldots, N_{p}-1 \\
\mathbf{u}_{k+i \mid k}=\mathbf{0}, \quad i=N_{u}, N_{u}+1, \ldots, N_{p}-1 \\
\mathbf{u}_{k+i \mid k} \in\{0,1\}^{n m} \\
\mathbf{X}_{\text {min }} \leqslant \mathbf{x}_{k+i \mid k} \leqslant \mathbf{X}_{\text {max }}, \quad i=1, \ldots, N_{p}-1
\end{array}\right.
$$

where $Q=Q^{T} \geqslant 0$ and $R=R^{T}>0$ are weighting matrices, $\mathbf{X}_{\min }$ and $\mathbf{X}_{\max }$ are the lower and upper energy capacity bounds, respectively, and the pair $\left(Q^{1 / 2}, A\right)$ is detectable.

Define an extended vector $\mathbf{x}=\left[\mathbf{x}_{k+1 \mid k}^{T}, \ldots, \mathbf{x}_{k+N_{p} \mid k}^{T}\right]^{T}$ that contains the predicted states involved in the optimization problem (6):

$$
\begin{aligned}
\mathbf{x} & =\Phi \mathbf{x}_{k \mid k}+\Gamma \mathbf{u} \\
\Phi & =\left[\begin{array}{c}
A \\
A^{2} \\
\vdots \\
A^{N_{p}}
\end{array}\right], \Gamma=\left[\begin{array}{cccc}
B & 0 & \cdots & 0 \\
A B & B & \cdots & 0 \\
\vdots & \vdots & \ddots & \vdots \\
A^{N_{p}-1} & A^{N_{p}-2} & \cdots & A^{N_{p}-N_{u}}
\end{array}\right]
\end{aligned}
$$

Then, the optimisation cost function is rewritten in a matrix form and (6) is described as a MIQP (see e.g. [13]):

$$
\begin{aligned}
& \arg \min _{\mathbf{u}} \mathbf{u}^{T} H \mathbf{u}+2 \mathbf{u}^{T} F \mathbf{x}_{k \mid k} \\
& \text { subject to: }\left\{\begin{array}{l}
\mathbf{u} \in\{0,1\}^{n m N_{u}} \\
\bar{F}_{i n_{x}} \Gamma \mathbf{u} \leqslant \bar{G}_{i n_{x}}-\bar{F}_{i n_{x}} \Phi \mathbf{x}_{k \mid k} \\
\bar{F}_{e q_{u}} \mathbf{u}=\bar{G}_{e q_{u}}
\end{array}\right.
\end{aligned}
$$

where $H=\Gamma^{T} \bar{Q} \Gamma+\bar{R}$ and $F=\Gamma \bar{Q} \Phi, \bar{Q}=\operatorname{diag}[Q, \ldots, Q]$, $\bar{R}=\operatorname{diag}[R, \ldots, R]$. The inequality and equality constraints (2), (3) and (5) on $\mathbf{x}_{k \mid k}$ and $\mathbf{u}_{k \mid k} \forall k$, are fully described by $\bar{F}_{i n_{x}} \in \mathbb{R}^{s \times n}, \bar{G}_{i n_{x}} \in \mathbb{R}^{s}, \bar{F}_{e q_{u}} \in \mathbb{R}^{p \times r}$ and $\bar{G}_{e q_{u}} \in \mathbb{R}^{p}$, $p=\left(N_{p}-N_{u}\right) n m, r=N_{p} n m, s=N_{p} q$.

It is worth mentioning that the degrees of freedom of the control design are related to the choice of the weighting matrices $Q$ and $R$, and the prediction $N_{p}$ and control $N_{u} \leqslant$ $N_{p}$ horizons.

\section{IMPLEMENTATION}

The control strategy described above is now implemented in a real-life test-bench in order to evaluate the proposed power management strategy and assess the efficiency of the controlled system. The hardware and software aspects of the test-bench are first shortly described. Then, implementation issues of the control approach are discussed. Lastly, the experimental results are pursued.

\section{A. Test-bench description}

The hardware test-bench considered here, with a sink, a router, and $n=6$ sensor nodes $S_{i}$, is shown in Figure 2. The sink is a laptop equipped for communication with a Wi-Fi card. The router allows data exchanges between the sink and the SNs. The SNs are connected with Flyport WiFi $802.11 \mathrm{~g}$ modules developed by openPicus [18]. The Flyport WiFi 802.11g module is a programmable system-on-module with integrated WiFi 802.11g connectivity. Its typical current consumption are shown in Table II. The energy battery level of the nodes can be measured. The sensing elements are temperature \& humidity sensors DHT-11 [19].

At time $k$, node $S_{i}$ can work in a unique mode. In the present case, $m=3$ functioning modes $M_{j}$ are defined. This choice is a trade-off between ensuring the system performance and the energy consumption minimization. It is similar to the choice in [20] and [21]. The modes are described as follows (see also Table III): 
TABLE II: Current consumption of different components in Flyport WiFi [18]

\begin{tabular}{|l||l|l|}
\hline Module & Current & Remarks \\
\hline $\begin{array}{l}\text { Wi-Fi not con- } \\
\text { nected }\end{array}$ & $39,75 \mathrm{~mA}$ & $\begin{array}{l}\text { MCU ON and Wi-Fi on but not } \\
\text { connected }\end{array}$ \\
\hline Wi-Fi connected & $162,70 \mathrm{~mA}$ & $\begin{array}{l}\text { MCU ON and Wi-Fi infrastructure } \\
\text { mode connected to an access point }\end{array}$ \\
\hline Wi-Fi burst & $282,50 \mathrm{~mA}$ & RF Burst on data TX \\
\hline Hibernate mode & $28,21 \mathrm{~mA}$ & $\begin{array}{l}\text { MCU ON and Wi-Fi transceiver } \\
\text { OFF }\end{array}$ \\
\hline Sleep mode & $1,44 \mathrm{~mA}$ & $\begin{array}{l}\text { MCU OFF and Wi-Fi transceiver } \\
\text { OFF }\end{array}$ \\
\hline
\end{tabular}

TABLE III: Functioning modes for node $S_{i}$

\begin{tabular}{lcccc}
\hline Mode & Processor & Radio & Sensor(s) & Battery monitor \\
\hline \hline$M_{1}$ & Active & Tx, Rx & On & On \\
\hline$M_{2}$ & Sleep & Off & Off & Off \\
\hline$M_{3}$ & Off & Off & Off & Off \\
\hline
\end{tabular}

- $M_{1}$ is the Active mode. In this mode, sensing, computing and communication SSs are "duty cycled" (see Figure 3), each SS being off by default and entering a wake-up mode periodically with a sampling period $T_{s}=1 \mathrm{~min}$ to sense, process and exchange data with the sink. Note that the duty cycle is itself split in smaller duty cycles, allowing more control of the node energy consumption in the Active mode. Figure 4 shows the typical current consumption of the SN working in mode $M_{1}$. The waveform corresponds to a wireless SN application cycle: the node is awake from the sleep state. It collects data and prepares the packets to be transmitted. Then, the packets are sent to the sink.

- $M_{2}$ corresponds to the Standby mode. The duty cycle of this mode is depicted in Figure 3. In the sleep state, only a small part of the processor is active, corresponding to the Real Time Clock (RTC) Quartz system. The RTC allows to wake up the $\mathrm{SN}$ each $T_{w}=1 h$ to receive the commands from the sink and monitor the battery remaining capacity.

- $M_{3}$ is the Faulty mode. During the network lifespan, some nodes may become unavailable (due to e.g. physical damage, lack of power resources $X_{0}^{i} \backslash X_{\max }^{i} \leq \delta$ ) or additional nodes might be deployed in the faulty $\mathrm{SN}$ state. The SN can exit from this mode when for instance, the battery is recharged by the harvesting system $\left(X_{0}^{i} \backslash X_{\max }^{i}>\delta\right)$ or the physical damages are repaired. $\delta$ is defined for each battery and depends on its characteristics.

\section{Mission definition}

For this test-bench, $n=6 \mathrm{SNs}$ have been deployed in a working office. In order to regulate the air conditioning system, temperature and humidity are sensed through the WSN. During the day, when the office is in use, a good quality of measures can be achieved with 3 SNs. During the night, $1 \mathrm{SN}$ is enough to sens the temperature and humidity in the office unused. Precisely, the mission is split in two

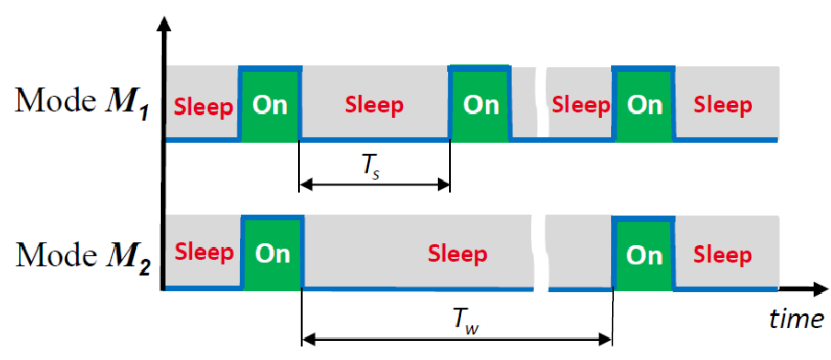

Fig. 3: Duty cycles of $M_{1}$ and $M_{2}$ modes

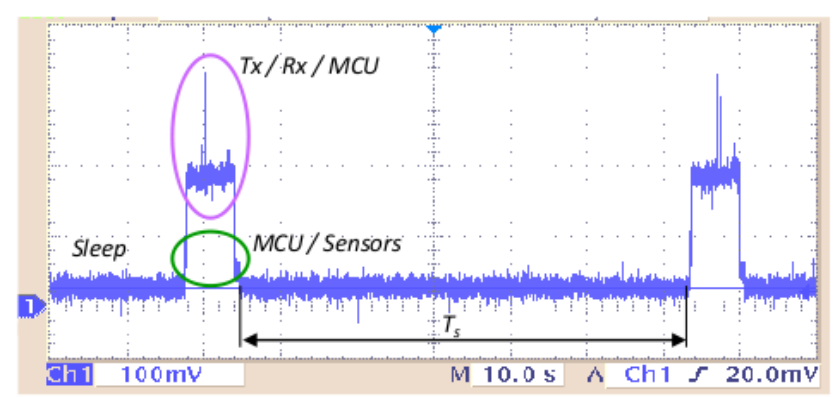

Fig. 4: Waveform of a cycle for our $\mathrm{SN}$ working in mode $M_{1}$ (current consumption measured with resistance of $1 \Omega$ )

phases corresponding respectively to day and night periods of time. Therefore, the constraints that define the mission have to be dynamically changed, depending on the time schedule, leading to a dynamic mission:

\begin{tabular}{cccc}
\hline \multicolumn{2}{c}{ Time period } & $d_{1}$ & Objectives \\
\hline \hline Day & $8 \mathrm{am}-6 \mathrm{pm}$ & 3 & 3 nodes in $M_{1}$ \\
\hline Night & $6 \mathrm{pm}-8 \mathrm{am}$ & 1 & 1 nodes in $M_{1}$ \\
\hline
\end{tabular}

Consider that at time instant $k_{0}$ all the SNs of the system are Active (in mode $M_{1}$ ). It is necessary to transmit their initial energy battery level and receive the control from sink. Then a sink checks whether that the node batteries have enough energy so that any node $S_{i}$ can fulfill the mission (i.e. being in mode $M_{1}$ ). If this is the case, during the day period, 3 nodes will by placed in mode $M_{1}$ while the $n-3$ others will by placed in $M_{2}$. And during the night period, 1 $\mathrm{SN}$ will by placed in mode $M_{1}$ and $n-1$ will by placed in mode $M_{2}$. As soon as the relative battery capacity of a SN is lower than $\delta$ or SN has other faulty, this SN will fall in mode $M_{3}$. Then, the control law assigns new modes to the remaining nodes in order to meet the dynamic mission while minimizing the energy consumption of the sensor network.

\section{B. Control application}

Two control methods can be applied when a sensor falls in mode $M_{3}$. First, an hybrid model can be considered to switch from one model to another when a node mode changes [22]. The second method is based on Fault-tolerant Control (FTC) approaches [23]. In this case, the set of constraints is modified, this second approach is used in the present paper.

For the system (1), $A=\mathbf{I}_{6}$ while the components of matrix $B$ are calculated from the values given in Table 
TABLE IV: Power consumption $B_{i j}(m A \cdot h)$ of node $S_{i}$ in mode $M_{j}$

\begin{tabular}{|c|c|c|c|}
\hline Sensor node & Mode $M_{1}$ & Mode $M_{2}$ & Mode $M_{3}$ \\
\hline \hline$S_{1}$ & 9.42 & 1.58 & 0 \\
\hline$S_{2}$ & 9.88 & 1.65 & 0 \\
\hline$S_{3}$ & 9.86 & 1.63 & 0 \\
\hline$S_{4}$ & 9.86 & 1.63 & 0 \\
\hline$S_{5}$ & 9.70 & 1.65 & 0 \\
\hline$S_{6}$ & 8.93 & 1.55 & 0 \\
\hline
\end{tabular}

TABLE V: SN battery characteristics and harvesting capability

\begin{tabular}{|c|c|c|c|l|}
\hline $\begin{array}{c}\text { Sensor } \\
\text { node }\end{array}$ & $\begin{array}{c}\text { Battery } \\
\text { Type }\end{array}$ & $\begin{array}{c}\text { Nominal } \\
\text { Voltage } \\
{[\mathbf{V}]}\end{array}$ & $\begin{array}{c}\text { Battery } \\
\text { capacity } X_{\text {max }}^{i} \\
{[\mathbf{m A} \cdot \mathbf{h}]}\end{array}$ & $\begin{array}{l}\text { Harvesting } \\
\text { availability } \\
E_{i i}[\mathbf{m A} \cdot \mathbf{h}]\end{array}$ \\
\hline \hline$S_{1}$ & LiPo & 3.7 & 1100 & missing \\
\hline$S_{2}$ & LiPo & 3.7 & 1100 & missing \\
\hline$S_{3}$ & LiPo & 3.7 & 1100 & 270 \\
\hline$S_{4}$ & LiPo & 3.7 & 950 & missing \\
\hline$S_{5}$ & LiPo & 3.7 & 950 & 270 \\
\hline$S_{6}$ & LiPo & 3.7 & 2300 & missing \\
\hline
\end{tabular}

IV, multiplied by the battery nominal voltage value of the corresponding SN (see Table V where the battery characteristics associated with each node are provided). Note that the numerical values are derived from Table II and lab. measurements. Table $\mathrm{V}$ also provides the initial capacity of the batteries associated with each SN. These latter numerical values are obtained from the technical data sheet of $\mathrm{Li}$ polymer rechargeable batteries [24] and solar cells [25].

The weighting matrices $Q$ and $R$ that appear in the definitions of $\bar{Q}$ and $\bar{R}$ in (7) are chosen equal to:

$$
Q=\mathbf{0}_{6 \times 6} ; \quad R=B^{T} \times\left(\left(R u^{T} \times R u\right) / 2\right) \times B
$$

where

$$
R u=\operatorname{diag}\left[\left[\begin{array}{ccc}
r u_{1} & 0 & 0 \\
0 & r u_{1} & 0 \\
0 & 0 & r u_{1}
\end{array}\right], \cdots,\left[\begin{array}{ccc}
r u_{6} & 0 & 0 \\
0 & r u_{6} & 0 \\
0 & 0 & r u_{6}
\end{array}\right]\right]
$$

and $r u_{i} \triangleq \min \left\{X_{\text {max }}^{i} / x_{k \mid k}^{i}\right\}, x_{k \mid k}^{i} \neq 0$. The choice $Q=$ $\mathbf{0}_{6 \times 6}$ lies in the fact that the state dynamics should evolve as slowly as possible [26]. The choice of $R$ implies a tradeoff between bigger energy consumption and smaller energy battery level for node penalization. This choice allows to keep the same battery power level in all SNs.

The inequality constraints (2) become:

$$
\underbrace{\left[\begin{array}{c}
\mathbf{I}_{6} \\
-\mathbf{I}_{6}
\end{array}\right]}_{F_{i n_{x}}}\left[\begin{array}{c}
x_{1} \\
\vdots \\
x_{6}
\end{array}\right] \leq \underbrace{\left[\begin{array}{llllll}
X_{\max }^{1} & \cdots & X_{\max }^{6} & 0 & \cdots & 0
\end{array}\right]^{T}}_{G_{i n_{x}}}
$$

while the equality constraints (3) and (5) are defined as:

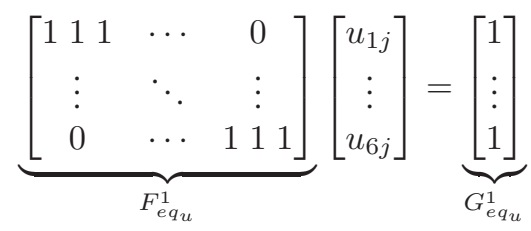

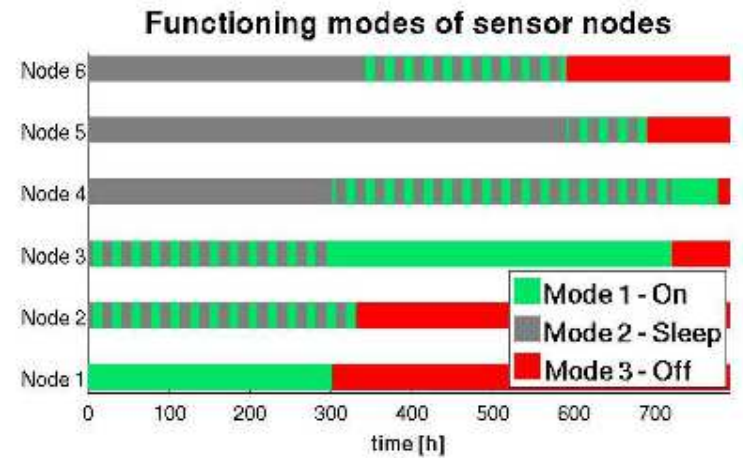

Fig. 5: Functioning modes of sensor nodes vs. time

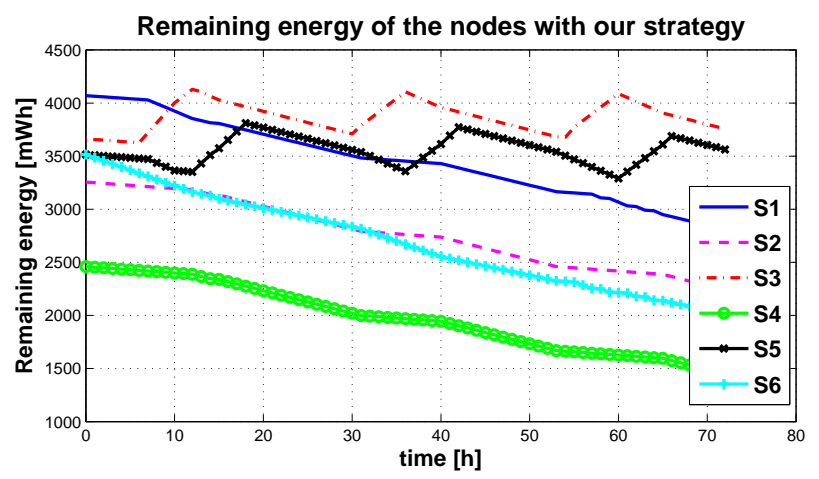

Fig. 6: Energy harvesting availability with harvesting

$$
\underbrace{\left[\begin{array}{llllllllll}
1 & 0 & 0 & 1 & 0 & 0 & \cdots & 1 & 0 & 0 \\
0 & 1 & 0 & 0 & 1 & 0 & \cdots & 0 & 1 & 0 \\
0 & 0 & 1 & 0 & 0 & 1 & \cdots & 0 & 0 & 1
\end{array}\right]}_{F_{e q_{u}}^{2}}\left[\begin{array}{c}
u_{1 j} \\
\vdots \\
u_{6 j}
\end{array}\right]=\underbrace{\left[\begin{array}{c}
a \\
b \\
c
\end{array}\right]}_{G_{e q u}^{2}}
$$

where $a$ and $b$ correspond to the number of nodes in modes $M_{1}$ and $M_{2}$, respectively. During daytime $a=3$ and at night $a=1$, see a mission definition. $c$ corresponds to the number of nodes fallen in mode $M_{3}$ with $c=n-a-b$. The other matrices are defined as follows: $F_{e q_{u}}=\left[\begin{array}{cc}F_{e q_{u}}^{1} & F_{e q_{u}}^{2}\end{array}\right]^{T} ; G_{e q_{u}}=$ $\left[\begin{array}{ll}G_{e q_{u}}^{1} & G_{e q_{u}}^{2}\end{array}\right]^{T} ; \bar{F}_{e q_{u}}=\operatorname{diag}\left[F_{e q_{u}}, \ldots, F_{e q_{u}}\right], \bar{G}_{e q_{u}}=$ $\operatorname{diag}\left[G_{e q_{u}}, \ldots, G_{e q_{u}}\right], \quad \bar{F}_{i n_{x}}=\operatorname{diag}\left[F_{i n_{x}}, \ldots, F_{i n_{x}}\right]$, $\bar{G}_{i n_{x}}=\operatorname{diag}\left[G_{i n_{x}}, \ldots, G_{i n_{x}}\right]$. The prediction and control horizons are chosen equal to $N_{p}=5, N_{u}=1$ respectively. As the considered system presents slow dynamics, these horizons are enough. The decision period (i.e. the time period when the power control is run) is $\Delta=T_{w}=1 \mathrm{~h}$. Thus, the MIQP problem is solved on-line at each decision time $k \Delta$.

The power control of the WSN considered is written in Python. The MIQP problem is solved with PICOS [27] using the Mosek solver [28]. Coordination between the SNs and sink is realized via the LINC coordination environment [29].

\section{Experimental Results}

The application results are provided in Figure 5 where for each $\mathrm{SN}$, its associated mode imposed by the proposed 


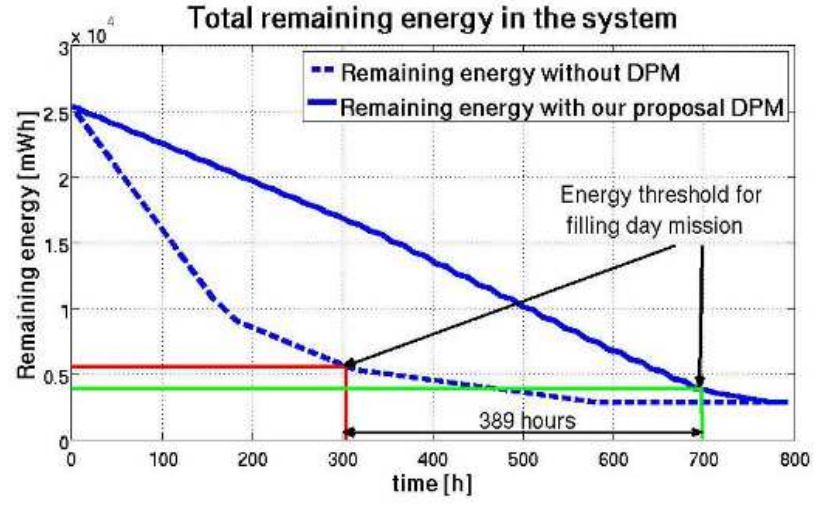

Fig. 7: Total energy evolution comparison (with and without our control strategy)

control strategy can be seen. The simulation lasts only two cycles $(2 \times 24$-hour $)$, but it is still enough to see the system evolution. The mission can be fulfilled at each time until at least 3 nodes do not have their batteries drained or have not faultily (i.e. a communication problem). The remaining battery energy states are presented in Figure 6. Note that some of the SNs have harvesting system as a solar cell. This explains the increase of the remaining energy level during a day period of time, when the sun is shining. Other harvesting profiles may lead to change the system lifespan and distribution of the Active SNs.

Figure 7 illustrates the comparison the total remaining energy in the system with our proposal control strategy and without it, i.e. with use the usual communication scheme. It is when each $\mathrm{SN}$ works in mode $M_{1}$. We can seen, that the proposed control expands the WSN lifespans by $95 \%$ with given harvesting capability of SNs compared to the usual scheme.

\section{COnClusions And Future Research}

Energy-efficiency is an important issue in WSNs, because battery resources are limited. Mechanisms that preserve the energy resources are highly desirable, as they have a direct impact on the network lifetime.

In this paper, a power consumption control strategy for a WSN has been proposed. The energy in the sensor nodes is modeled using a linear state-space representation. Harvesting capability of the SNs is also taken into account. The WSN has to provide a given functionality (named the mission), expressed with a set of constraints. The control problem is defined as a MIQP one that imposes a unique functioning mode to each SN at each decision time. Implementation results in a real test-bench show the efficiency of the proposed control method. Power savings in the SNs, and in the entire WSN, of more than $95 \%$ were possible compared to without control algorithm, when all sensor nodes are in Active mode.

\section{REFERENCES}

[1] Ian F Akyildiz, Weilian Su, Yogesh Sankarasubramaniam, and Erdal Cayirci. Wireless sensor networks: a survey. Computer networks, 2002.
[2] Nagendra Prasad Mandru. Optimal power management in wireless sensor networks for enhanced life time. Journal of Global Research in Computer Science, 3:73-78, 2012.

[3] Fred Chen, Fabian Lim, Omid Abari, Anantha Chandrakasan, and Vladimir Stojanovic. Energy-aware design of compressed sensing systems for wireless sensors under performance and reliability constraints. IEEE Transactions on Circuits and Systems I: Regular Papers, 2013.

[4] Wei Hailong, Shen Yan, and Wei Tuming. Dynamic power management of wireless sensor networks based on grey model. In Advanced Computer Theory and Engineering (ICACTE), 2010 3rd International Conference on. IEEE, 2010.

[5] Vinod Sharma, Utpal Mukherji, Vinay Joseph, and Shrey Gupta. Optimal energy management policies for energy harvesting sensor nodes. Wireless Communications, IEEE Transactions on, 2010.

[6] Cesare Alippi, Giuseppe Anastasi, Mario Di Francesco, and Manuel Roveri. Energy management in wireless sensor networks with energyhungry sensors. Instrumentation \& Measurement Magazine, IEEE, 2009.

[7] Daniel E Quevedo, Jan Ostergaard, and Anders Ahlen. Power control and coding formulation for state estimation with wireless sensors. Control Systems Technology, IEEE Transactions on, 2014.

[8] Xue Jun Li, Shao Xuguang and other. Application of model predictive control in wireless sensor networks. In Infor., Communications and Signal Processing (ICICS) 2011 8th Inter. Conf. on. IEEE, 2011.

[9] Tom La Porta, Chiara Petrioli, and Dora Spenza. Sensor-mission assignment in wireless sensor networks with energy harvesting. In Sensor, Mesh and Ad Hoc Communications and Networks (SECON), 2011 8th Annual IEEE Communications Society Conf. on. IEEE, 2011.

[10] Olesia Mokrenko, Suzanne Lesecq, Warody Lombardi, Diego Puschini, Carolina Albea, and Olivier Debicki. Dynamic power management in a wireless sensor network using predictive control. In Industrial Electronics Society, IECON 2014-40th Annual Conference of the IEEE. IEEE, 2014.

[11] David Q Mayne, James B Rawlings, Christopher V Rao, and Pierre OM Scokaert. Constrained model predictive control: Stability and optimality. Automatica, 2000.

[12] Eduardo F Camacho and Carlos Bordons Alba. Model predictive control. Springer, 2013.

[13] Jan Marian Maciejowski. Predictive control: with constraints. Pearson education, 2002.

[14] Frank Allgöwer and Alex Zheng. Nonlinear model predictive control. Birkhäuser Basel, 2000.

[15] Alberto Bemporad and Manfred Morari. Predictive control of constrained hybrid systems. In Nonlinear model predictive control. Springer, 2000.

[16] Alberto Bemporad and Manfred Morari. Control of systems integrating logic, dynamics, and constraints. Automatica, 1999.

[17] Vivek Mhatre and Catherine Rosenberg. Homogeneous vs heterogeneous clustered sensor networks: a comparative study. In IEEE International Conference on Communications, 2004. IEEE, 2004.

[18] http://www.openpicus.com.

[19] http://www.aosong.com/en/products/details.asp?id=109.

[20] Paulo Sérgio Sausen, José Renato de Brito Sousa, Marco Aurélio Spohn, Angelo Perkusich, and Antônio Marcus Nogueira Lima. Dynamic power management with scheduled switching modes. Computer Communications, 31, 2008.

[21] Vijay Raghunathan, Saurabh Ganeriwal, and Mani Srivastava. Emerging techniques for long lived wireless sensor networks. Communications Magazine, IEEE, 44, 2006.

[22] Jan Lunze and Françoise Lamnabhi-Lagarrigue. Handbook of hybrid systems control: theory, tools, applications. Cambridge University Press, 2009.

[23] Eduardo F Camacho, Teodoro Alamo, and David Muñoz de la Pena. Fault-tolerant model predictive control. In Emerging Technologies and Factory Automation (ETFA), 2010 IEEE Conference on. IEEE, 2010.

[24] http://www.farnell.com/datasheets/1666650.pdf and 1666648.pdf.

[25] https://hackspark.fr/fr/1-5w-solar-panel-81x137.html.

[26] Robert L Williams, Douglas A Lawrence, et al. Linear state-space control systems. John Wiley \& Sons, 2007.

[27] Picos: A python interface for conic optimization solvers, http://picos.zib.de. 2012.

[28] http://www.mosek.com/.

[29] Maxime Louvel and François Pacull. Linc: A compact yet powerful coordination environment. In Coordination Models and Languages. Springer, 2014. 